\title{
El ministerio transmedia: expansiones narrativas y culturas participativas ${ }^{1}$
}

\author{
Carlos A. Scolari ${ }^{2}$ \\ María-José Establés ${ }^{3}$
}

Recibido: 2017-07-16

Enviado a pares: 2017-07-24
Aprobado por pares: 2017-07-30

Aceptado: 2017-08-01

DOI: 10.5294/pacla.2017.20.4.7

Para citar este artículo / to reference this article / para citar este artigo

Scolari CA, Establés MJ. (2017). El ministerio transmedia: expansiones narrativas y culturas participativas. Palabra Clave 20(4), 1008-1041. DOI: 10.5294/pacla.2017.20.4.7

\section{Resumen}

Este artículo analiza uno de los fenómenos más destacados de la industria cultural española de la segunda década del siglo XXI: El Ministerio del Tiempo. A los pocos días de su estreno, esta serie — nacida como producto "monomediático" estrictamente televisivo- se transformó en uno de los ejemplos más interesantes de narrativa transmedia producidos en español. En concreto, el artículo se focaliza en dos aspectos clave para comprender las dinámicas que pueden generar las narrativas transmedia. Por un lado, las tensiones que se han producido por las bajas audiencias televisivas de la serie (que siempre ponen en jaque su continuidad) y la enorme actividad de sus fans en internet, un conflicto que apunta a las formas de medición ( $y$ al concepto mismo) de audiencia en el post-broadcasting. Y por otro, el fenómeno emergente en el contexto español a partir de El Ministerio del Tiempo: la profesionalización de los fans, la cual se evidencia, por ejemplo, en la venta de productos — realizados por los usuarios - en la misma web de Ra-

\footnotetext{
1 Este artículo ha sido financiado por el Ministerio de Economía, Industria y Competitividad del Gobierno de España a través del programa Ayudas para la Formación de Doctores 2015 (Ref. BES-2015-071455).

2 orcid.org/0000-0002-7792-0345. Universitat Pompeu Fabra. carlosalberto.scolari@upf.edu

3 orcid.org/0000-0001-9674-3981. Universitat Pompeu Fabra. mariajose.estables@upf.edu
} 
dio Televisión Española. El artículo se cierra con algunas reflexiones sobre el carácter disruptivo de la serie y otras cuestiones vinculadas a un mundo narrativo que dejará su marca en la historia de la industria cultural española más allá del corto plazo.

\section{Palabras clave}

Transmedia storytelling; televisión pública; fandom; post-broadcasting; fan labor; profesionalización de los fans (Fuente: Tesauro de la Unesco). 


\section{The Transmedia Ministry: Narrative Expansions and Participatory Cultures}

\section{Abstract}

This article analyzes one of the most outstanding phenomena of the Spanish cultural industry of the second decade of the 21 st century: The Department of Time. A few days after its release, this series-born as a strictly televised "monomeditic" product-became one of the most interesting examples of transmedia narrative produced in Spanish. In particular, this article focuses on two key aspects to understand the dynamics that transmedia narratives can generate. On the one hand, the stress caused by the low TV ratings of the series (which always threaten its continuity) and the enormous online activity of their fans, a conflict that points to the forms of measurement (and the concept itself) audience in post-broadcasting. And on the other, the emerging phenomenon in the Spanish context from The Department of Time: the fans' professionalization, which is evidenced, for example, in the sale of products - by users - on the same website of Radio Televisión Española. The article ends with some reflections on the disruptive nature of the series and other issues linked to a narrative world that will leave its mark on the history of the Spanish cultural industry beyond the short term.

\section{Keywords}

Transmedia storytelling; public television; fandom; post-broadcasting; fan labor; fan professionalization (Source: Unesco Thesaurus). 


\section{0 ministério transmídia: expansões narrativas e culturas participativas}

\section{Resumo}

Este artigo analisa um dos fenômenos mais destacados da indústria cultural espanhola da segunda década do século XXI: $O$ ministério do Tempo. Aos poucos dias da sua estreia, esta série — nascida como produto "monomediático" estritamente televisivo - transformou-se em um dos exemplos mais interessantes de narrativa transmídia produzidos em espanhol. Em concreto, $\mathrm{o}$ artigo tem seu foco em dois aspectos chave para compreender as dinâmicas que podem gerar as narrativas transmídia. Por um lado, as tensões produzidas pelas baixas audiências da série na televisão (que sempre põem em jogo a sua continuidade) e a enorme atividade de seus fãs na internet, um conflito que aponta às formas de medição (e o próprio conceito) de audiência no pós-broadcasting. E por outro lado, o fenômeno emergente no contexto espanhol a partir de $O$ ministério do Tempo: a profissionalização dos fãs, fator evidenciado, por exemplo, na venda de produtos — realizados pelos usuários - no próprio site web da Rádio Televisão Espanhola. O artigo é concluído com algumas reflexões sobre o caráter disruptivo da série e outras questões vinculadas a um mundo narrativo que deixará a sua marca na história da indústria cultural espanhola mais além do curto prazo.

\section{Palavras-chave}

Transmedia storytelling; televisión pública; fandom; post-broadcasting; fan labor; profissionalização dos fãs (Fonte: Tesauro da Unesco). 


\section{Una serie de culto}

A pesar de ser una producción muy reciente, El Ministerio del Tiempo (2015) ha despertado un fuerte interés, no solo en la crítica televisiva y los medios de comunicación, sino también en la comunidad científica. Antes de que finalizara la primera temporada, surgieron estudios académicos que ponían en valor aspectos formales y de contenido de la serie, así como su repercusión en las comunidades de fans y en la crítica de televisión especializada.

Una de las primeras obras colectivas que se ha publicado es el libro Dentro de 'El Ministerio del Tiempo', coordinado por Concepción Cascajosa Virino (2015), texto donde se explora la serie desde distintos puntos de vista (narrativo, estudios de género, estudios de cultura de fans, historiográfico, etc.) y encontramos trabajos centrados en el estudio de las narrativas transmedia, audiencias y fans (Marcos, 2015; Scolari, 2015b; Establés-Heras y Rivera-Pinto, 2015; Meléndez-Malavé y Sedano-Amundarain, 2015; Varona Aramburu y Lara, 2015). Cascajosa Virino también ha publicado otros dos artículos, uno en 2016 y otro en 2017 que firma junto con Molina Cabañate. En el primero de ellos, trata la cuestión de la investigación sobre audiencias televisivas tomando como caso de estudio esta serie, mientras que en el segundo los investigadores exploran las características del universo transmediático de El Ministerio del Tiempo.

De hecho, la cuestión de las narrativas transmedia y el fandom de la serie son dos de los aspectos que más interesan a los investigadores que están estudiando la serie. En este sentido, Crisóstomo (2016) explora la capacidad de los fans, tanto refiriéndose a los elementos constructivos de series como El Ministerio del Tiempo, como a sus personajes y al ámbito de la narrativa transmedia. Siguiendo esta temática, se enmarcan los trabajos de MirandaGalbe y Figuero-Espadas (2016) y el artículo de Establés-Heras (2016). Miranda-Galbe y Figuero-Espadas (2016) argumentan que las aportaciones de los prosumidores tienen la misma importancia que las oficiales de la serie, ya que "hacen que ésta crezca y sea más completa, generando una fidelidad por parte de los espectadores mucho mayor que las series que solo se preocupan por su desarrollo monomediático” (p. 15). Así, Establés-Heras 
(2016) analiza, por un lado, el rol de los fans como generadores de contenidos que expanden un universo narrativo transmediático de dos series ( $E l$ Ministerio del Tiempo e Isabel), y por otro, el debate surgido a causa de las posibles cancelaciones de la serie de El Ministerio del Tiempo, en el cual el fandom reflexiona sobre el papel que debe desempeñar una televisión pública como garante de la producción de programas y ficciones de calidad.

Por otra parte, debido a la importancia que la serie le otorga al conocimiento de la historia de España, otros académicos están centrando sus análisis en aspectos más relacionados con esa disciplina. Así, cabe destacar otros trabajos como el de Rueda Laffond y Coronado Ruiz (2016), en el que analizan, desde una perspectiva crítica, la combinación de recreación histórica, ciencia ficción y discurso de la memoria a través de los capítulos de la serie y de las creaciones de los fans, y también el artículo de Rodríguez Mateos y Hernández-Pérez (2015), en el cual ponen de manifiesto los nuevos roles del documentalista audiovisual en la generación de valor en los productos audiovisuales, en el análisis de las audiencias diferidas y en la reutilización del patrimonio audiovisual de las cadenas.

El presente artículo, que forma parte del trabajo de investigación de tesis doctoral, se centra en analizar la construcción y el despliegue de los mundos narrativos transmedia más allá de las clásicas ficciones producidas en los Estados Unidos y presta particular atención a las producciones y prácticas de los fans en el contexto de una cultura colaborativa (Jenkins, 2006), así como a la reconceptualización de la medición de audiencias en el contexto mediático actual. Los objetivos del artículo son los siguientes:

- Describir y analizar el mundo narrativo de El Ministerio del Tiempo.

- Describir y analizar algunas prácticas y creaciones de fans que pueden ser consideradas casos de profesionalización de este tipo de producción.

- $\quad$ Reflexionar sobre las tensiones que El Ministerio del Tiempo ha generado dentro del ecosistema de medios español, en el contexto de 
una transición de un sistema centrado en el broadcasting a otro centrado en las redes.

Nuestro análisis difiere del resto de estudios previos realizados que centran los análisis en el rol de los fans, no solo como posibles creadores profesionales de productos mediáticos, sino también en su papel a la hora de cuestionar las mediciones de las audiencias de series, que pueden ser consideradas cult TV (Hills, 2004), ya que cuentan con un público especializado y minoritario. Por ello, consideramos que El Ministerio del Tiempo cumple con las tres definiciones que destaca Hills sobre lo que es la cult $T V(2004$, pp. 509-510):

1. Las series definidas como cult $T V$ suelen ser de los géneros de ciencia ficción, horror o fantasía. Algunos casos paradigmáticos son Doctor Who, Buffy the Vampire Slayer, Xena, the Warrior Princess o Charmed. En el caso de El Ministerio del Tiempo, se puede denominar una serie de ciencia ficción y fantasía con tintes de ficción histórica.

2. Existen análisis de la serie a través de textos e "intertextos" tanto en ámbitos periodísticos especializados como en académicos. En los párrafos anteriores, hemos destacado algunos de los análisis académicos de la serie, si bien en el ámbito periodístico nos encontramos reportajes en revistas de $s c i-f i$, en las secciones de televisión de periódicos diarios de tirada nacional o en páginas web de medios de comunicación especializados en televisión y cine.

3. El Ministerio del Tiempo es una cult TV, porque se define por el análisis crítico de las prácticas y los textos producidos por y para fans de la serie, así como la "devoción” del fandom que es la que le confiere el halo de televisión de culto. En el caso de El Ministerio del Tiempo, las comunidades de fans están desempeñando un rol de devotos hacia su objeto de culto. Incluso el nombre con el que se autodenominan, ministéricos, refleja en cierta medida esa obsesión por el mundo narrativo de la serie. 


\section{"El Ministerio del Tiempo"como narrativa transmedia}

\section{¿Qué es una narrativa transmedia?}

Según el relato oficial compartido por académicos y profesionales, el concepto de narrativa transmedia (transmedia storytelling) fue introducido por Henry Jenkins en un artículo publicado en Technology Review en 2003, en el cual afirmaba: "Hemos entrado en una nueva era de convergencia de medios que vuelve inevitable el flujo de contenidos a través de múltiples canales”. Según Jenkins (2003),

Ios niños que han crecido consumiendo y disfrutando Pokémon en varios medios esperan la misma experiencia de El Ala Oeste de la Casa Blanca a medida que se hacen mayores. Pokémon se despliega a través de juegos, programas de televisión, películas y libros, y ningún medio se privilegia sobre el otro.

Las nuevas generaciones de consumidores se han vuelto cazadoras y recolectoras de información, les gusta sumergirse en las historias, reconstruir el pasado de los personajes y conectarlos con otros textos dentro de la misma franquicia.

No cuesta mucho reconstruir un canon de obras transmedia, desde mundos narrativos nacidos en el cine, como Star Wars o Indiana Jones, hasta franquicias que tuvieron su origen en el cómic (Superman, Ironman, Spiderman), pasando por producciones de matriz televisiva $(24$, Lost) o videolúdica (Final Fantasy, Resident Evil, Assassins Creed). Cuando Star Wars pasó al mundo impreso,

sus novelas expandieron la línea temporal para mostrar eventos nunca vistos en las trilogías cinematográficas, o difundieron historias de personajes secundarios como en la serie Tales of the Cantina, donde se conocieron con más detalle esos curiosos alienígenas que en la película original quedaban de fondo. Cuando Star Wars llegó a los

4 Las traducciones son nuestras. 
videojuegos, esos juegos no repitieron los eventos de las películas, sino que nos mostraron cómo era la vida de un entrenador Jedi o de un cazador de recompensas. (Jenkins, 2003)

Desde esta perspectiva, cada medio hace un aporte a la construcción del mundo narrativo transmedia. Evidentemente, las aportaciones de cada medio o plataforma de comunicación difieren entre sí. Tal como explica Jenkins (2003), en las narrativas transmedia, cada medio

hace lo que mejor sabe hacer: una historia puede ser introducida en un largometraje, expandirse en la televisión, novelas y cómics, y este mundo puede ser explorado y vivido a través de un videojuego. Cada franquicia debe ser lo suficientemente autónoma para permitir un consumo autónomo. 0 sea, no debes ver la película para entender el videojuego, y viceversa.

En un texto posterior, Jenkins (2006, p. 46) reafirmará que las narrativas transmedia son "historias contadas a través de múltiples medios. En la actualidad, las historias más significativas tienden a fluir a través de múltiples plataformas mediáticas”. En este contexto, es el productor transmedia (transmedia producer) el encargado de diseñar la estrategia transmedia, determinar qué parte del relato se expresa en cada medio - explotando al máximo sus posibilidades semióticas de expresión-y crear los espacios para que los fans den rienda suelta a su imaginación.

Dicho en otros términos: las narrativas transmedia son una particular forma del relato que se expande a través de diferentes medios y plataformas de comunicación y van mucho más allá de la simple "adaptación" de un medio a otro: la historia que cuenta el videojuego no es la misma que aparece en el cine o en la novela.

El otro componente fundamental de las narrativas transmedia viene dado por las creaciones de los fans, los cuales no dudan en enriquecer esos mundos narrativos con nuevas historias en todo tipo de soporte mediático. El fenómeno del fan fiction existe desde el mismo origen de la cultura de masas (o incluso antes), pero las nuevas plataformas colaborativas lo han puesto en el centro de la escena. Las viejas audiencias televisivas o 
cinematográficas, al igual que los lectores tradicionales de cómics o novelas, se conformaban con consumir su producto favorito y, en el mejor de los casos, aspiraban a montar un club de fans para festejar a sus personajes o autores preferidos (Jenkins, 1992; Busse y Hellekson, 2006). Algo ha cambiado en las últimas décadas, sobre todo desde la llegada de los procesos de digitalización y la difusión de la World Wide Web: algunos consumidores se convirtieron en prosumidores (productores más consumidores), se apropiaron de sus personajes favoritos y expandieron aún más sus mundos narrativos. Según Jenkins, esta es la otra característica que define las narrativas transmedia: los usuarios cooperan activamente en el proceso de expansión transmedia. Ya sea escribiendo una ficción y colgándola en fanfiction. net, ya sea grabando una parodia y subiéndola a YouTube, los prosumidores del siglo XXI son activos militantes de las narrativas que les apasionan.

Entonces, ¿qué es una narrativa transmedia? Es el canon más el fandom. En pocas palabras: en las narrativas transmedia, la producción oficial (cubierta por el derecho de autor y que busca el lucro) se complementa con la producción de los fans (a menudo al margen de las leyes del copyright y sin objetivos comerciales). Como señala Jenkins (2006), estamos frente a dos mundos - la industria de los medios y las culturas colaborativas - con lógicas opuestas, pero condenados a mantener intercambios entre ellas. Las narrativas transmedia nacen ahí, donde al mismo tiempo (como sostiene el título del libro de Jenkins [2006]) las culturas convergen y los viejos y nuevos medios colisionan.

\section{Mundos narrativos transmedia}

Las narrativas transmedia, más que crear un relato lineal, proponen mundos narrativos (storyworlds) que abarcan diferentes medios/plataformas y experiencias de producción/consumo. Esta dispersión textual que encuentra en lo narrativo su hilo conductor — aunque sería más adecuado hablar de una red de personajes y situaciones que conforman un mundo- es uno de los aspectos más genuinos de la cultura de masas contemporánea. Inspiradas en los estudios sobre los cibermundos creados a partir de los videojuegos, investigadoras como Klastrup y Tosca (2004) han introducido el concepto de mundo transmedial (transmedial world). 
Los mundos transmediales son sistemas de contenido abstractos a partir de los cuales se puede actualizar o derivar un repertorio de historias y personajes de ficción a través de una variedad de medios y plataformas de comunicación. Lo que caracteriza a un mundo transmedial es que la audiencia y los creadores comparten una imagen mental de la "mundanidad" (worldness), eso es, una serie de rasgos distintivos de ese universo. Esta idea de la "mundanidad" proviene principalmente de la primera versión presentada de ese mundo, pero puede ser reelaborada y transformada a lo largo del tiempo, ya sea por sus mismos creadores, ya sea con la complicidad de sus consumidores, ahora convertidos en prosumidores (Klastrup y Tosca, 2004, p. 409).

Un mundo transmedial (transmedial world) difiere de la narrativa transmedia (transmedia storytelling) de Jenkins en que la segunda se centra en la narración concreta de una historia a través de diferentes medios, mientras que el primero trata de las propiedades abstractas que recorren y atraviesan las diferentes manifestaciones de ese mundo. Según Klastrup y Tosca, los elementos centrales de los mundos transmediales son los siguientes:

- Mŷthos: hace referencia a los conflictos y las batallas del mundo, que incluyen los personajes del mundo, por ejemplo la creación de la Tierra Media y las diferentes razas detalladas por Tolkien en The Lord of the Rings.

- Topos: indica la configuración del mundo en un periodo histórico y una geografía específica, por ejemplo la Tierra Media, sus lenguas, su poesía y tradiciones en The Lord of the Rings.

- Ethos: hace referencia a la ética explícita e implícita del mundo y el código moral de comportamiento que los personajes del mundo deben seguir, por ejemplo la lucha del bien contra el mal o la exaltación de la amistad en The Lord of the Rings (2004, pp. 409-410).

Estas tres propiedades de los mundos transmediales resultan de gran utilidad para el análisis de las estrategias desplegadas por sus creadores. Con estos apuntes teóricos y categorías analíticas, podemos pasar a la segunda fase: la descripción y el análisis del mundo narrativo de El Ministerio del Tiempo. 


\section{Expansiones transmedia}

El 24 de febrero de 2015 el canal La 1 de la televisión pública de España (RTVE) estrenó una nueva serie titulada El Ministerio del Tiempo. Esta serie fantástica creada por los hermanos Javier y Pablo Olivares relata las aventuras de un grupo de agentes de un ministerio secreto que controla una serie de puertas que permiten viajar a través de la historia española. Si bien la función del Ministerio es mantener sin cambios la historia de España, los agentes no tardarán en descubrir que existen tanto excepciones a la regla como agentes dobles que apuestan por cambiar la historia del país. De hecho, en El Ministerio del Tiempo está presente una reflexión crítica sobre la complejidad, los conflictos y las contradicciones de la cultura e historia españolas. Además, se trata de una producción plagada de referencias intertextuales (Kristeva, 1978; Bajtin, 1989; Genette, 1982) —quizá fue este hecho el que ha favorecido la creación de una gran complicidad con los espectadores - que explora todo tipo de género televisivo, desde la comedia al drama, pasando por el relato policial, el thriller, la ciencia ficción o la épica histórica.

En la actualidad, El Ministerio del Tiempo es una de las series españolas más premiadas y reconocidas en el ámbito internacional, y cuenta ya con un remake oficial en Portugal (Ministério do Tempo), e incluso ha mantenido un litigio con la serie norteamericana Timeless, debido a una demanda por un presunto plagio a El Ministerio del Tiempo (Patten, 2017).

La primera temporada de la serie consta de ocho episodios y tuvo una audiencia media de 2.5 millones de espectadores, es decir, una cuota de pantalla correspondiente a $12.3 \%$ (Pimentel, 2015). En unas pocas semanas, y gracias a la actividad en las redes sociales, la serie generó una enorme comunidad de fans, no solo en España, sino también en países de América Latina. Pocos días después de su lanzamiento, los primeros contenidos generados por los fans, desde carteles hasta juegos de mesa, páginas del Ministerio en Facebook, remixes (por ejemplo los personajes de $E l$ Ministerio del Tiempo en versión The Sims o The Simpsons) y otras expresiones de fanart, aparecieron en las redes sociales y rápidamente se pusieron en circulación, con lo cual realimentaron y expandieron aún más la comunidad de fans crecida alrededor de la serie. 
La difusión oficial de la serie se centró en la creación de un community manager, Aurelio Pimentel (un personaje ficticio del siglo XIX que comienza a conocer el mundo digital del siglo XXI) a través de una amplia campaña en redes sociales (Twitter, Facebook, Instagram y WhatsApp, principalmente), así como acciones puntuales en Tumblr o Telegram. Además, había contenidos adicionales en la web y en el foro oficial de RTVE, y algunos cebos en varios programas televisivos de La 1 y La 2, como en los informativos, o en programas de entretenimiento como MasterChefo Saber y Ganar.

Sin embargo, a partir de la segunda temporada (cuya emisión comenzó el 24 de febrero de 2016), los productores de El Ministerio del Tiempo decidieron convertirla en una experiencia narrativa transmedia en los términos definidos en las secciones precedentes. Además de los doce episodios televisivos, en la segunda temporada, el mundo narrativo de El Ministerio del Tiempo incluyó un episodio especial filmado en realidad virtual, una serie de podcasts, una novela y una presencia más coordinada en las redes sociales. Al equipo de producción se sumó Pablo Lara, un productor transmedia, que aparecía mencionado como tal en los créditos de cada episodio.

El episodio especial filmado en realidad virtual titulado El tiempo en tus manos fue una producción pionera en el contexto español e internacional: se trata de la primera expansión de una serie televisiva a través de contenidos inmersivos que deben ser consumidos con lentes 3D. El tiempo en tus manos, una producción de 10 min de duración grabada con un sistema de estereoscopía, permite visitar las instalaciones del Ministerio e interactuar con los personajes.

Por otra parte, la ausencia de uno de los actores por motivos profesionales (se encontraba grabando otra serie) llevó a la creación de una serie de podcasts que mantuvieron abierto un hilo narrativo paralelo ambientado en la guerra de Filipinas (1896-1898). La novela El tiempo es el que es (Schaaff y Pascual, 2016) expande el mundo narrativo de la televisión con tres nuevas aventuras: una al siglo VIII para rescatar a un agente perdido en esa época, otra al siglo XVII ambientada en Cartagena de Indias y otra para salvar a una compañera que ha sido atrapada por los nazis durante la 
Segunda Guerra Mundial. Todas estas iniciativas se complementaron con una fuerte presencia en las redes sociales (Twitter, Facebook, WhatsApp e Instagram), una webserie donde el personaje Angustias (interpretado por Francesca Piñón) se confiesa frente a la cámara, la Intranet del Ministerio del Tiempo, un portal web con decenas de contenidos extras (fotografías, vídeos o melodías para teléfonos móviles) y una tienda oficial de productos de mercadotecnia, en la cual profundizaremos más adelante.

Ante el inicio de la tercera temporada, que comenzó a emitirse el 1 de junio de 2017, se ha seguido apostando por el mantenimiento de algunas acciones de difusión (las campañas en redes sociales, los contenidos adicionales en la web oficial o nuevos episodios de la webserie de Angustias), pero también por la ampliación del universo transmedia de la serie. Así, los productores han querido unir las dos temporadas televisivas a través del cómic Tiempo al Tiempo (El Torres, Bressend, Martínez y Molina, 2017), en el que la patrulla protagonista tiene que salvar la vida al subsecretario del Ministerio del Tiempo. Además, se han creado nuevos podcasts ambientados en la década de 1960 para incorporar de nuevo a un personaje que había dejado la serie, y se ha editado un juego de mesa basado en la ficción.

Por otra parte, La 1 vuelve a retomar el uso de aplicaciones de doble pantalla que tanto éxito le reportaron en series históricas como Isabel, si bien con mejoras técnicas muy significativas. Por eso, durante la emisión de los episodios, ya sea en directo, ya sea en diferido, se puede sincronizar la aplicación móvil El Ministerio del Tiempo Dive y profundizar así en la descripción de las tramas, los lugares y los personajes históricos, e incluso conocer curiosidades de la producción de la serie. Asimismo, los usuarios pueden añadir contenidos adicionales que no aparezcan en la aplicación y que puedan ser de interés para el resto de espectadores. De esta forma, se sigue reforzando el carácter didáctico de la serie, pero sin perder su esencia lúdica y de entretenimiento.

Además, tras el parón de mitad de temporada (midseason), se estrenará una nueva experiencia de realidad virtual más ambiciosa que la realizada durante la segunda temporada, que se llamará Salva el Tiempo, en la cual 
los usuarios podrán interactuar con personajes de El Ministerio del Tiempo (Prensa RTVE, 2017).

\section{"El Ministerio del Tiempo" como mundo narrativo transmedia}

Para terminar esta descripción del mundo narrativo transmedia de El Ministerio del Tiempo, y antes de pasar a la descripción y análisis de la actividad de los fans de la serie, conviene detenerse en las características de esta construcción mediático-narrativa. El Ministerio del Tiempo es un excelente ejemplo de narrativa transmedia, tal como la entiende Jenkins (2003, 2006): se trata de un relato formado por una variedad de textos, cada uno de los cuales actúa como puerta de ingreso a la narrativa. La historia de El Ministerio del Tiempo se cuenta a través de muchos medios y plataformas, y los fans participan de manera activa en la expansión narrativa y, en ese caso en particular, han llegado incluso a garantizar la continuidad del mundo narrativo al presionar para que se renovara la serie televisiva. Dicho en términos de Jenkins (2006), en El Ministerio del Tiempo, se produce la tan citada convergencia entre la industria de los medios y las culturas colaborativas.

Si se analiza el mundo narrativo de El Ministerio del Tiempo desde la perspectiva de Klastrup y Tosca (2004), el investigador se encuentra con la siguiente construcción narrativa:

- Mŷthos: el mundo de El Ministerio del Tiempo está integrado por una serie de personajes que mantienen relaciones muy ricas entre sí que se van reformulando a medida que pasan los episodios. La figura del agente doble ha servido en varias ocasiones para complejizar esas relaciones y abrir nuevos filones narrativos. Si bien existe una gran batalla por cambiar/preservar el orden temporal, la trama de cada episodio se enriquece con muchos otros conflictos secundarios, desde triángulos amorosos hasta cuestiones paterno-filiales o luchas por el poder dentro de la estructura ministerial.

- Topos: El Ministerio del Tiempo parte de un espacio-tiempo fijo (la sede del Ministerio en Madrid durante el año de emisión) y se ex- 
pande en cada episodio con misiones a lo largo de la historia y geografía española. Los agentes pueden terminar operando en Toledo durante el reinado de los Reyes Católicos, en Barcelona en la Segunda Guerra Mundial, en Sevilla durante el Siglo de Oro, o en Madrid durante la invasión francesa a comienzos del siglo XIX. El Ministerio del Tiempo es una serie que aprovecha la experiencia y know-how de la televisión pública española para recrear escenografías y vestuarios de épocas pasadas. Y cuando alguna de estas reconstrucciones no están realizadas a la perfección, son los fans los primeros en indicar los errores en los foros y las redes sociales.

- Ethos: como ya se mencionó, la existencia de agentes dobles y de personajes que intentan cambiar el pasado en su propio beneficio plantea permanentes dilemas dentro de El Ministerio del Tiempo. Uno de los personajes sufre la pérdida de su pareja en un accidente y cada vez que puede se escapa al pasado para volver a verla con vida. En uno de los episodios más dramáticos (el último de la primera temporada), los personajes conocen a Federico García Lorca y, a pesar de estar al tanto de su trágico destino, deben regresar a 2015 sabiendo que será fusilado durante la guerra civil y ellos no podrán hacer nada al respecto.

Como se puede observar, El Ministerio del Tiempo es una obra que detrás de la aparente sencillez de su trama admite muchos niveles de lectura, los cuales se enriquecen con otros aspectos - como la ya mencionada intertextualidad, un tema que no abordaremos en este artículo-y la expansión del relato en muchos medios y plataformas con la colaboración de sus fans. A esta actividad que emerge de las redes sociales y foros de discusión en línea, está dedicada la tercera sección de este artículo.

\section{Más allá del canon}

\section{TEl post-broadcasting y la medición de audiencias}

Además de la experiencia transmedia que caracteriza a la serie, uno de los debates que ha surgido en torno a El Ministerio del Tiempo está vinculado a la tensión que ha generado este producto dentro del ecosistema de medios español, el cual se encuentra en un contexto de transición de un sistema 
centrado en el broadcasting a otro hegemonizado por el consumo mediático a demanda y en red.

A pesar de la gran acogida por los fans, que se autodenominan ministéricos, esta ficción lleva lidiando prácticamente desde sus inicios con la sombra de su posible cancelación. La primera vez que los fans sospecharon sobre el probable final de la serie fue tras la emisión del segundo capítulo de la primera temporada (Tiempo de Gloria), debido a un inesperado cambio de fecha y hora de la emisión del episodio. ${ }^{5}$ Por ello, en todas las acciones llevadas a cabo durante las siguientes semanas, los fans se organizaron de forma coordinada para pedir la renovación de la serie a través de las redes sociales, siguiendo el ejemplo de otras campañas similares, como la de la serie estadounidense Fringe (Guerrero-Pico, 2017). Es la primera vez que el fandom de una serie española realiza una campaña de esta índole, lo cual convierte a los ministéricos en un colectivo de fans comprometidos, no solo para expandir el mundo narrativo de esta ficción, sino para mantenerlo en antena. Por tanto, El Ministerio del Tiempo es uno de los primeros ejemplos españoles de cult TV (Hills, 2004).

Pero esa no sería la única vez que desde la cadena de televisión se modificaran el día y la hora de emisión. ${ }^{6}$ Como cabría esperar, esto afectaría a la audiencia tradicional que representa al público que se sienta frente al televisor para ver en directo la serie. Sin embargo, esa pérdida relativa de televidentes se compensaría durante las dos primeras temporadas a través de los visionados en directo y en diferido en la web de RTVE.es. De todas las series que ofrece TVE en internet, El Ministerio del Tiempo es la más vista a

5 El primer episodio se emitió el martes 24 de febrero de 2015 a las 22.45 horas. Sin embargo, La 1 decidió cambiar la fecha de emisión a los lunes a las 22.15 horas para evitar competir con la audiencia de la serie Bajo Sospecha (Antena 3) y el programa Levántate (Telecinco), ya que el estreno de la serie cosechó un menor número de espectadores del que estaba previsto.

6 Otro ejemplo en el cambio de fecha y día se produjo el 16 de marzo de 2015. La 1 decidió emitir en su desconexión territorial de Andalucía a la hora de la serie, el debate de los candidatos en las elecciones autonómicas. Este hecho movilizó todavía más a los fans a través del hashtag \#AndaluciaQuiereMDT4. Finalmente, los andaluces tuvieron la opción de ver la serie, o bien en directo a través de RTVE.es, o bien esperaron a verla frente al televisor una vez finalizado el debate. La controversia fue mayor, teniendo en cuenta que el cuarto episodio contaba con el crossover del personaje de la reina Isabel la Católica, gracias a un cameo de la actriz Michelle Jenner, protagonista de la exitosa serie de La 1, Isabel. 
través de RTVE.es (Pimentel, 2015). Además, durante la emisión de todos los episodios, la serie ha tenido una gran acogida en Twitter, tras lo cual se ha convertido en trending topic en el mundo, tanto los hashtags oficiales de los capítulos como algunos personajes históricos y de la cultura popular. En concreto, la medición en Twitter de los ocho episodios de la primera temporada fue de 353850 usuarios, 3687500 impresiones y 7187 autores únicos que sumaron 21255 tuits (Pimentel, 2015).

Durante la emisión de los últimos episodios de la primera temporada, los fans se encontraron con la incertidumbre de si existiría una nueva temporada de El Ministerio del Tiempo, ya que aún no estaba confirmada la realización de una segunda. Ese fue el detonante del nacimiento de la primera campaña para salvar a la serie de la cancelación, que se gestó a través del grupo oficial de becarios en WhatsApp de El Ministerio del Tiempo.

Así, en el caso de la primera temporada, el 10 de marzo de 2015 apareció el hashtag \#TVErenuevaMdT. La campaña de salvación de la serie no contaba desde sus inicios con unos protocolos muy rígidos de actuación, es decir, no existía un decálogo como tal de qué acciones se tenían que llevar a cabo. No obstante, los mensajes iniciales se enviaron y coordinaron desde las cuentas de Twitter de los fans miembros del exclusivo grupo de WhatsApp (Establés-Heras y Rivera-Pinto, 2015). Los tipos de tuits que se enviaron eran de tres tipos: animar a los fans a unirse a la campaña, poner en valor contenidos y personajes que aparecían en la serie y reivindicar que en la televisión pública se emitan productos televisivos de calidad. En menos de dos horas, el lema fue acogido con entusiasmo, ya que se convirtió en trending topic y a él se unieron periodistas, fans, directores de cine, actores, etc. (figura 1).

Además de las acciones en Twitter, los fans promovieron una campaña para salvar la serie en la plataforma change.org, que alcanzó las 5031 firmas, o enviaron correos electrónicos en los cuales solicitaban la renovación al Defensor del Espectador de TVE. Una vez lanzada la campaña de salvación de la serie, los fans contaron también con la complicidad del equipo técnico y artístico de la serie para que la renovación llegara a buen puerto. 


\section{Figura 1. Algunos tuits enviados por los fans que lanzaron la campaña de renovación.}

\begin{tabular}{|c|c|}
\hline $\begin{array}{l}\text { A las 12:30 comenzamos a Tweetear con } \\
\text { \#TVErenuevaMdT para que @rtve renueve la } \\
\text { serie de 'El Ministerio del Tiempo' } \\
\text { (@MdT_TVE). \#MdT3 } \\
\text { 12:12 - } 10 \text { mar } 2015\end{array}$ & $\begin{array}{l}\text { Para todos los seguidores de } \\
\text { \#EIMinisterioDelTiempo, es hora de presionar } \\
\text { para que renueven esta joya. Este es el } \\
\text { hashtag \#TVErenuevaMdT } \\
\text { 12:16 - } 10 \text { mar. } 2015\end{array}$ \\
\hline $\begin{array}{l}\text { Petición a todos los fans del } \\
\text { \#MinisterioDelTiempo : usad este hashtag } \\
\text { \#TVErenuevaMdT si quereis una segunda } \\
\text { temporada! } 6 \text { @MdT_TVE } \\
\text { 12:28-10 mar:2015 }\end{array}$ & $\begin{array}{l}\text { La calidad de los guiones, actores, } \\
\text { fotografía, banda sonora... es un lujazo } \\
\text { tenerlo en la tv pública @tve_tve @MdT_TVE } \\
\text { \#TVErenuevaMdT } \\
\text { 12:40 - } 10 \text { mar. } 2015\end{array}$ \\
\hline $\begin{array}{l}\text { Con \#EIMinisterioDelTiempo @MdT_TVE la } \\
\text { televisión pública española se ha puesto en } \\
\text { vanguardia \#TVErenuevaMdT } \\
\text { 12:18 - } 10 \text { mar. } 2015\end{array}$ & $\begin{array}{l}\text { Por una tv pública de calidad, la serie } \\
\text { @MdT_TVE tiene que ser renovada @tve_tve } \\
\text { \#TVErenuevaMdT } \\
\text { 12:31 - } 10 \text { mar. } 2015\end{array}$ \\
\hline
\end{tabular}

Fuente: Twitter.

En este sentido, las acciones que se siguieron llevando a cabo se continuaron coordinando a través de WhatsApp y otras plataformas. Durante dos semanas no pararon de enviar mensajes a las redes sociales Twitter y Facebook. La campaña finalizó cuando finalmente RTVE anunció la renovación para una nueva temporada el 24 de marzo de 2015.

La segunda temporada de El Ministerio del Tiempo se estrenó el 15 de febrero de 2016. A pesar del esfuerzo invertido en la implementación de la estrategia transmedia de la serie, los resultados de las audiencias tradicionales de la segunda temporada no lograron superar a la primera: El Ministerio del Tiempo alcanzó una audiencia promedio de 2.3 millones de espectadores, en concreto $11.9 \%$ de cuota de pantalla (Oterino, 2016). Este rendimiento en las audiencias no puede ser aislado de la frenética actividad de los fans de la serie en las redes, donde cada episodio nuevo de El Ministerio del Tiempo se convirtió, semana tras semana, en trending topic durante y después de su emisión. De hecho, los fans intensificaron el ruido social en redes como Twitter y Facebook. Nació así otro nuevo hashtag denominado \#RenovacionMdT, que se lanzó el 9 de abril de 2016, tras la emisión del cuarto capítulo de la segunda temporada (El Monasterio del Tiempo). 
Por otra parte, durante la emisión de esta temporada, los fans volvieron a mostrar su faceta más combativa, ya que los datos de las audiencias tradicionales no auguraban una posible renovación. En esta ocasión, además de reactivar los hashtags en Twitter, una vez finalizada la temporada, los fans retransmitieron a través de redes sociales un capítulo ficticio imaginado por ellos a través del hashtag\#TiempoDeChupitos (Pimentel, 2016).

Al finalizar la segunda temporada (23 de mayo 2016), la historia volvió a repetirse: RTVE no había confirmado la continuación de la serie y, de nuevo, los fans siguieron manteniendo viva la campaña para la renovación contractual. Tras el éxito de la primera campaña, los fans contaban con la experiencia previa y aprovecharon su capacidad de movilización para seguir haciéndose escuchar. ${ }^{7}$ De esta forma, pasaron de la campaña de 2015, que era más improvisada e inicialmente ideada por un grupo reducido de fans a través de WhatsApp y Twitter, a la de 2016, más elaborada y coordinada desde distintas plataformas digitales y llevada a cabo por varios grupos de fans (creadores de podcasts, comunidades en Facebook, grupos en WhatsApp y tuiteros).

En este caso, la respuesta de RTVE se hizo esperar cinco meses, pero, finalmente, la serie fue renovada por una tercera temporada en septiembre de 2016. Una de las consecuencias de esta espera ha sido la modificación de algunas de las tramas argumentales ideadas para la última temporada, ya que los creadores no pudieron contar con todos los miembros del elenco artístico inicial (López, 2017; Migelez, 2017; La Vanguardia, 2016).

La tercera temporada, al igual que la segunda, cuenta con trece episodios y un parón de mitad de temporada. Hasta la fecha de redacción de este artículo, los seis primeros episodios correspondientes a la primera mitad de la tercera temporada han tenido una media de audiencia de 1582000

7 Además de la denuncia que interpuso la productora Onza Entertainment, una de las productoras de El Ministerio del Tiempo, por el presunto plagio de Timeless, las similitudes entre una y otra serie se han manifestado en distintas áreas. Cabe reseñar que el creador de Timeless, Eric Kripke, animó, a través de un tuit a los fans de la serie, a que se movilizaran para evitar la cancelación de esta (Bohannon, 2017). La forma de solicitar la renovación y, sobre todo, el énfasis que le puso para salvar la serie antes de que finalizara la temporada, recuerdan las dos campañas para la renovación de El Ministerio del Tiempo (en este caso lideradas por los fans y no por el showrunner de la serie). 
espectadores, es decir, $10.1 \%$ de cuota de pantalla (Ver Tele, 2017), unos datos inferiores a los de las dos temporadas previas.

Pero, además, la emisión de la nueva temporada está siendo distinta de las dos anteriores. Una vez se emita el último capítulo en La 1 a finales de 2017, la serie se podrá ver en 190 países a través de la plataforma de streaming Netflix (De Pablos, 2016). La entrada de Netflix ha supuesto una mejora tanto en el presupuesto de producción de la serie como en su distribución internacional. Sin embargo, también ha tenido como consecuencia que El Ministerio del Tiempo se haya dejado de emitir en la web de RTVE fuera de las fronteras españolas de forma gratuita, dado que Netflix requiere una suscripción de pago para el visionado de sus contenidos. Esta medida ha supuesto el malestar de muchos fans que seguían la serie, principalmente desde países de América Latina, por lo cual algunos de ellos han decidido consumirla a través de descargas no autorizadas disponibles en distintas webs.

Si bien el debate entre la medición dela audiencia tradicional ylas audiencias sociales y en diferido ha sido muy intenso durante las dos primeras temporadas de la serie, en el caso de la tercera, la situación se ha visto sustancialmente modificada por la entrada de Netflix en la producción de la ficción, puesto que la financiación ha dejado de ser íntegramente pública para ser mixta (públicoprivada). Hasta la segunda temporada, la serie era financiada por RTVE, yla producción la llevaban a cabo las productoras Cliffhanger y Onza Entertainment. En este sentido, en 2015 y 2016, en el trasfondo de las campañas de renovación, no solo se encontraba el deseo de los fans por mantener en antena $E l$ Ministerio del Tiempo, sino también la reivindicación de que un canal público de televisión estatal no debe competir con los canales privados para conseguir la mayor cuota de pantalla en horario de prime time, sino que debe primar la calidad de sus contenidos, independiente del número de espectadores que tenga según las mediciones tradicionales. Siguiendo esta consideración, unos días antes de la renovación de la segunda temporada de la serie, surgió el hashtag \#ConteoVisionadoMdT_TVE que hace referencia a esta situación, y en el que se destaca que RTVE no está financiada por bloques publicitarios, como es el caso de las televisiones generalistas privadas, 
por lo cual reclamaban así el visionado real de la serie a través de plataformas digitales, como es la propia web de RTVE.

Sin embargo, en 2017, si bien el debate surgido en torno a la calidad de los contenidos para los canales de televisión públicos se ha mantenido, la discusión sobre el rol que debe mantener RTVE se ha visto modificado por el factor Netflix. Netflix es una empresa, de titularidad privada, que aunque tiene sistemas de conteo reales tiene como política no mostrarlos públicamente. El argumento con la reivindicación que hacían los fans de El Ministerio del Tiempo para pedir el conteo real del visionado de la serie pierde peso con esta nueva coproducción de la serie. Si bien muchos fans acogieron con entusiasmo la entrada de Netflix en la serie, lo cual suponía un aumento en el presupuesto de producción y la renovación por la tercera temporada, ahora están descubriendo la contraprestación de que la serie deje de ser de emisión pública a $100 \%$ : cuota de suscripción para ver la serie entera una vez se emita la tercera temporada en España y tan solo se podrán ver cinco episodios de dicha temporada simultáneamente en la web de RTVE.es (Terán, 2017). Además, la demanda del conteo real tanto en directo como en diferido en el portal web de RTVE pierde fuelle, ya que la serie no está disponible para su visionado fuera de España, y dentro de las fronteras nacionales, no se pueden ver todos los episodios. De hecho, se está conjugando un nuevo modelo de producción, circulación y consumo en la industria de medios española, que reclama nuevos espacios para productos audiovisuales innovadores como El Ministerio del Tiempo.

Previamente, se han realizado algunas experiencias de consumo de series españolas en diferentes medios, principalmente en cines, como es el caso de Los Protegidos o Isabel, pero el caso de El Ministerio del Tiempo está yendo más allá (acuerdos entre plataformas de streaming y televisiones públicas, capítulos de la serie con realidad virtual, etc.). Por eso, podemos destacar que El Ministerio del Tiempo es uno de los pocos ejemplos de serie española que se encuentra dentro de la era del post-broadcasting del contexto televisivo español, ya que su consumo se realiza en distintos medios y plataformas, y que entra en confrontación con el modelo de broadcasting dominante en la industria mediática del país. 


\section{Procesos de profesionalización y prácticas del fandom ministérico}

No se puede hablar de El Ministerio del Tiempo sin hacerlo de sus fans. Los ministéricos son protagonistas del impacto social que ha tenido la serie en múltiples espacios físicos y digitales. Aunque nos encontremos inmersos en una clara expansión de los fandoms a través de redes sociales, la explotación de los espacios físicos es una de las señas de identidad de la serie. Por ello, no debe extrañar encontrarse a un grupo de personas hacerse fotografías en la puerta del Ministerio del Tiempo, que en realidad es el edificio Duquesa de Sueca, perteneciente al Ayuntamiento de Madrid, y que se encuentra ubicado en la plaza del Duque de Alba. Este espacio de peregrinaje de los fans (Reijnders, 2011) ha sido el punto de partida para que un grupo de fans haya creado distintas rutas turísticas que recorran las calles y los rincones de distintas ciudades que aparecen en la serie (Barcelona, Madrid, Sevilla o Alcalá de Henares). Estas quedadas ministéricas se suman a otros eventos organizados por fans, que plantean el revisionado de capítulos, partidas de rol y juegos de mesa, ya que son un complemento a los eventos organizados desde RTVE (los preestrenos de las temporadas o la presentación de productos oficiales de la serie).

A estas prácticas en espacios físicos se le suman la infinidad de manifestaciones en redes sociales, foros, blogs y páginas web. En concreto, la producción fan es muy prolífica (Lacalle, Castro y Castro, 2016): carteles, juegos de rol, cosplay, perfiles que imitan a los personajes en distintas redes sociales, remixes, fan arts, fanfics, fanvids, etc. Incluso, algunos de estos trabajos de los fans están repercutiendo en el ámbito de la innovación educativa (Establés-Heras, 2016). En relación con el ámbito del fanfic, una de las prácticas habituales de los fans de esta serie es la creación de capítulos en Twitter a través de micro-fanfics. Dos de los casos más paradigmáticos se han llevado a cabo entre el parón de las temporadas segunda y tercera: \#TiempoDeChupitos (cuando aún no se había renovado la serie) y \#TiempoDeEmisión (cuando La 1 todavía no había confirmado qué día se emitiría el primer capítulo de la tercera temporada). Ambos hashtags se convirtieron en trending topics mundiales. 
Gracias a este fandom tan comprometido, en la segunda temporada de la serie, los productores decidieron ampliar el universo transmedial a través de una tienda virtual de merchandising oficial con productos creados a demanda. Una vez que se agotaba un producto, ya no se realizaban más copias; el objetivo era promover la exclusividad de los productos y dotar a sus compradores, en cierta medida, de un estatus de coleccionista.

Para estudiar esta iniciativa, no se puede dejar de lado en el análisis las distintas perspectivas teóricas sobre esta cuestión, dependiendo de su mayor o menor grado de perspectiva crítica: fanworks como explotación (Jones, 2014; Hills, 2013; Fuchs, 2013), los derechos de autor de los productores y de los fans (De Kosnik, 2009) o cómo los fans desarrollan estos procesos creativos de hibridación con las industrias creativas (Roig, 2013). En este caso, los productos fueron diseñados por un grupo de ocho fans y los beneficios se repartieron entre ellos y los productores de la serie (Prensa RTVE, 2016). A través de esta experiencia pionera, que rompe moldes en el ámbito de difusión de una serie española, se pueden empezar a explorar posibles procesos y estrategias de profesionalización de los fans.

Para nuestro análisis, hemos escogido los productos que a fecha de junio de 2017 no están ya disponibles al público porque ya se han agotado. En ese sentido, hemos detectado que los productos más demandados son:

- $\quad$ Los textiles (sudadoras, camisetas y bolsas de tela): en este caso, las sudaderas más solicitadas son las diseñadas por Joe Tanaka, que es un Big Name Fan (Driscoll, 2006), es decir, uno de los principales fans creadores más reputados dentro del fandom ministérico. En el caso de los distintos tipos de camisetas, fueron diseñados por Conrado Martín, Jessica Gómez y Kiko Sánchez (su dibujo, que es el único en el que no aparecen personajes, se ha vendido impreso en bolsas de tela y libretas). Sobre la temática de los textiles, predomina la personificación de los personajes, en concreto, la patrulla de funcionarios protagonista de la primera temporada de la serie.

- Tazas y libretas: en relación con las libretas, la más solicitada fue la diseñada por Diego Cobo, que tiene como motivos principales los ros- 
tros de personajes de la serie. Las menos demandadas son en las que destacan mapas o el logo del Ministerio del Tiempo. En el caso de las tazas, se repite el mismo esquema: la más demandada tiene como temática a personajes de la serie (fue diseñada por Elena Mellado).

- Pósteres: a pesar de ser una de las manifestaciones de fan art más compartidas por los fans en redes sociales, no hemos encontrado ninguno de ellos que haya agotado sus ventas. De cada capítulo de la segunda temporada, se realizaron dos versiones de pósteres diferentes, una diseñada por Mikel Navarro y la otra por Sergio Iniesta.

Si bien tradicionalmente han existido tensiones entre los fans y las industrias culturales, en el caso de El Ministerio del Tiempo la apuesta ha sido de colaboración. Los productores han favorecido, en la medida de lo posible, una relación horizontal con sus fans, con la intención de salvar cuestiones controvertidas, como la de los derechos de autor, que en el caso de otras franquicias ha llegado a terminar en los tribunales de justicia.

Para algunos de los fans que han participado en esta tienda, más allá del volumen de ventas de sus productos, el hecho de haber formado parte de ella es su carta de presentación para conseguir trabajos profesionales en otros medios, principalmente en la industria editorial (revistas, libros, etc.). En este sentido, aunque en la tercera temporada la tienda no ha lanzado nuevos productos creados por fans, en el caso de los dibujantes de fan arts, las cuentas oficiales en redes sociales de El Ministerio del Tiempo están promocionando las obras que, semana tras semana, crean en relación con los nuevos capítulos que se van estrenando.

Como hemos mencionado en la segunda sección, en la última temporada los productos oficiales que expanden el mundo transmedial de la serie son el cómic y un juego de mesa: ambos cuentan entre sus creadores con fans de la ficción. Sin embargo, los nuevos productos no se han presentado como productos "exclusivos", sino que se pueden comprar en otros establecimientos convencionales, como tiendas de libros o de juegos de mesa, o también en grandes superficies comerciales. 
De hecho, no todos los procesos de profesionalización que se están desarrollando dentro del fandom ministérico se están manifestando en el seno de la tienda oficial. También se pueden encontrar multitud de fans que venden sus productos (desde un libro sobre curiosidades históricas relacionadas con la serie hasta muñecos creados en tela) o que los ofrecen de forma gratuita (diversos fanfics que aparecen en plataformas como fanfiction. net o en los blogs de los autores). Si bien excede los límites de este artículo, una futura línea de investigación sería el análisis a fondo de las motivaciones, estrategias y técnicas que llevan a cabo los fans a través de estas prácticas de incipiente profesionalización.

Otra de las prácticas habituales de los fans de El Ministerio del Tiempo está centrada en aspectos transnacionales y transculturales. Se trataría, por ejemplo, de acciones como el subtitulado de la serie con el objetivo de darle difusión en otros países, donde también se pueden encontrar distintas estrategias y procesos de profesionalización relacionados con la mejora de sus competencias interpretativas, tanto en el caso de estudiantes de esta disciplina como de traductores aficionados o titulados (Establés y Guerrero-Pico, 2017).

\section{El Ministerio del Tiempo: entre el broadcasting y el networking}

Después de este recorrido sobre el mundo narrativo transmedial de El Ministerio del Tiempo, llegó la hora de extraer algunas conclusiones y plantear interrogantes con vistas a futuras investigaciones en este terreno. Las narrativas transmedia son uno de los fenómenos más interesantes que emergen de la nueva ecología mediática (Scolari, 2012; Scolari, 2015a), se encuentran en plena fase de eclosión y, por este motivo, no resulta fácil ponerlas a foco para poderlas investigar con una cierta calma.

La ecología de los nuevos medios es fundamental para comprender el éxito de las estrategias transmedia. En el nuevo paradigma, el público está más que fragmentado: se atomizan en muchas pantallas, dispositivos, medios y plataformas. El modelo de negocio de la radiodifusión, basado en tener millones de espectadores sentados frente a la pantalla y viendo el mismo 
espectáculo, ya no es sostenible. En este contexto, la narración transmedia es una estrategia muy útil para construir nichos muy leales de consumidores alrededor de un mundo narrativo. El consumidor ve el producto audiovisual, compra el cómic, se sumerge en el videojuego o juego de mesa y visita el parque temático de su programa favorito. Si en la vieja ecología las audiencias estaban centradas en los medios (media-centered audiences), en la actual se centran en la narrativa (narrative-centered audiences).

En este contexto, El Ministerio del Tiempo es una producción que evidencia con toda claridad las tensiones que emergen en la transición de una ecología mediática centrada en el broadcasting a otra centrada en las redes. El Ministerio del Tiempo es una de las primeras obras españolas que se presenta como cult TV (Hills, 2004) y como exponente del post-broadcasting, fase en la cual se va configurando un nuevo modelo de producción, circulación y consumo en la industria de medios.

Por una parte, El Ministerio del Tiempo es el típico relato monomedia que por presiones del entorno comienza a expandirse y a colonizar otros medios y plataformas con la complicidad de los fans. En otras palabras: como muchísimos otros productos televisivos, desde la serie 24 (Fox) hasta Lost (ABC) o Águila Roja e Isabel (La 1) (Scolari, Guerrero-Pico y Establés, en prensa), El Ministerio del Tiempo pasó de ser una experiencia pensada para un único medio que, en una segunda fase y como reacción al entorno, terminó convirtiéndose en transmedia (Scolari, Jiménez y Guerrero, 2012; Scolari, 2013; Scolari, Guerrero-Pico y Establés, en prensa). Por estos motivos, se puede considerar El Ministerio del Tiempo un ejemplo de narrativa transmedia táctica frente a otros proyectos que fueron concebidos desde el inicio como una propuesta transmedia (narrativa transmedia estratégica).

La narrativa transmedia es un espacio fundamental para identificar y analizar las tensiones, los conflictos y las hibridaciones que surgen cuando, como dijo Jenkins (2006), "los viejos y los nuevos medios chocan”. En el caso de El Ministerio del Tiempo, es interesante ver que en 2016 el sitio web oficial de RTVE comenzó a vender distintos productos creados por los fans. Como se ha indicado, las relaciones entre el canon (industria de los medios de co- 
municación) y el fandom (cultura participativa) no son simples y pueden incluir hibridaciones y apropiaciones recíprocas (Bourriaud, 2002; Scolari, 2013; Guerrero-Pico y Scolari, 2016), pero también en procesos de explotación del trabajo de los fans (Jones, 2014; Hills, 2013; Fuchs, 2013). Por ello, en este contexto, pueden surgir distintas tensiones, pero también puntos de encuentro en los fans y las industrias mediáticas. De estas relaciones, pueden emerger nuevas formas de profesionalización que deberán ser tenidas en cuenta para futuras investigaciones en los ámbitos de la economía política de la comunicación y los estudios sobre fans. El rol de los fans, ya no solo como consumidores y prescriptores de contenidos, sino también como productores de calidad, deberá ser tenido en cuenta por la industria a la hora de diseñar nuevas estrategias de captación de talentos y también para la creación de productos mediáticos innovadores.

\section{Referencias}

Bajtin, M. (1989). Teoría y estética de la novela. Madrid: Taurus.

Bohannon, A. M. (2017). NBC's 'Timeless' faces cancellation as fans and celebrities rally to save it. En MoviePilot.com. Recuperado de https: / / moviepilot.com/p/timeless-cancelled-fans-celebs/4194433

Bourriaud, P. (2002). Postproduction: Culture as screenplay: How art reprograms the world. Nueva York: Lukas \& Sternberg.

Busse, K. y Hellekson, K. (2006). Introduction. Work in Progress. En K. Hellekson y K. Busse (eds.), Fan fiction and fan communities in the age of the internet (pp. 5-40). Jefferson, NC: McFarland Press.

Cascajosa Virino, C. (2015). Dentro de "El Ministerio del Tiempo". Madrid: Léeme Libros.

Cascajosa Virino, C. (2016). Buscando al espectador serial desesperadamente: la nueva investigación de audiencias y la serie El Ministerio del Tiempo. Dígitos, revista de comunicación digital, 2, 53-69. 
Cascajosa Virino, C. y Molina Cañabate, J. P. (2017). Narrativas expandidas entre la tradición y la innovación: construyendo el universo transmedial de El Ministerio del Tiempo. Tropelías. Revista de Teoría de la Literatura y Literatura Comparada, 27, 120-135.

Crisóstomo, R. (2016). "Fannibals ministéricos": el poder del "fandom". En Index Comunicación, 6(2), 77-86.

De Kosnik, A. (2009). Should fan fiction be free? Cinema Journal, 48(4), 118-124.

De Pablos, E. (2016). Netflix, spanish pubcaster RTVE pact on TV series 'The Department of Time'. En Variety.com. Recuperado de http:// variety.com $/ 2016 / \mathrm{tv} /$ global/netflix-rtve-the-department-of-time-1201950253/

Driscoll, C. (2006). One true pairing: The romance of pornography and the pornography of romance. En K. Hellekson y K. Busse (eds.), Fan fiction and fan communities in the age of the internet (pp. 97-114). Jefferson, NC: McFarland Press.

Establés-Heras, M. J. (2016). Entre fans anda el juego: audiencias creativas, series de televisión y narrativas transmedia. Revista Opción, $32(11), 476-497$.

Establés, M. J. y Guerrero-Pico, M. (en prensa). Los fans como traductores y distribuidores de contenido en el ecosistema transmedia: promocionando series de televisión españolas en el extranjero. En S. Torrado, J. G. Ferreras y G. Ródenas (eds.), Territorios transmedia y narrativas audiovisuales. Barcelona: UOC.

Establés-Heras, M. J. y Rivera-Pinto, D. (2015). “Ministéricos” en Twitter y WhatsApp, o cómo el poder de los fans puede mover montañas. En C. Cascajosa Virino (ed.), Dentro de "El Ministerio del Tiempo" (pp. 211-218). Madrid: Léeme Libros. 
El Torres, Bressend, D., Martínez, J. y Molina, S. (2017). Tiempo al tiempo. Girona: Aleta Ediciones.

Fuchs, C. (2013). Social media and capitalism. En T. Olsson (ed.), Producing the internet, critical perspectives of social media. Götemborg: Nordicom.

Genette, G. (1982). Palimpsestes: La littérature au second degré. París: Éditions du Seuil.

Guerrero-Pico, M. (2017). \#Fringe, audiences and fan labor: Twitter activism to save a TV show from cancellation. International Journal of Communication, 11, 1-22.

Guerrero-Pico, M. y Scolari, C. A. (2016). Narrativas transmedia y contenidos generados por los usuarios: el caso de los crossovers. Cuadernos.info, 38, 183-200.

Hills, M. (2004). Defining cult TV: Texts, inter-texts and fan audiences. En R. C. Allen y A. Hill (eds.), The television studies reader (pp. 509523). Londres: Routledge.

Hills, M. (2013). Fiske's 'textual productivity' and digital fandom: Web 2.0 democratization versus fan distinction. Participations, 10(1), 130-153.

Jenkins, H. (1992). Textual poachers: Television fans \& participatory culture. Nueva York: Routledge.

Jenkins, H. (2003). Transmedia storytelling: Moving characters from books to films to video games can make them stronger and more compelling. En Technology Review. Recuperado de https: //www.technologyreview.com/s/401760/transmedia_storytelling/

Jenkins, H. (2006). Convergence culture: When old and new media collide. Nueva York: NYU Press. 
Jones, B. (2014). Fifty shades of exploitation: Fan labor and Fifty Shades of Grey. Transformative Works and Cultures, 15.

Klastrup, L. y Tosca, S. (2004). Transmedial worlds-rethinking cyberworld design. Ponencia presentada en Cyberworlds, 2004 International Conference on, IEEE, Los Alamitos, CA.

Kristeva, J. (1978). Semiótica (vol. 1). Madrid: Espiral Fundamentos.

Lacalle, C., Castro, D. y Castro, M. (2016). España: innovación y tradición. En G. Orozco Gómez y M. I. Vasallo de Lopes (eds.), (Re)invención de géneros y formatos de la ficción televisiva (pp. 295-333). Porto Alegre: OBITEL.

La Vanguardia (12 diciembre 2016). Rodolfo Sancho abandona 'El Ministerio del Tiempo'. Recuperado de http://www.lavanguardia.com/ series/20161212/412566732043/rodolfo-sancho-deja-el-ministerio-del-tiempo.html

López Aroca, M. (2017). Javier Olivares: "Que se hable a la vez de Twin Peaks y de mi serie me ruboriza”. En Generación Fénix. Recuperado dehttp://generacionfenix.com/javier-olivares-creador-del-el-ministerio-del-tiempo-que-se-hable-la-vez-de-twin-peaks-de-mi-serie-ruboriza/

Marcos, N. (2015). Una serie para la generación Twitter. En C. Cascajosa Virino (ed.), Dentro de 'El Ministerio del Tiempo' (pp. 199-201). Madrid: Léeme Libros.

Meléndez Malavé, N. y Sedano Amundarain, J. A. (2015). Los administradores del tiempo: un análisis de los grupos de fans de Facebook. En C. Cascajosa Virino (ed.), Dentro de 'El Ministerio del Tiempo' (pp. 219-226. Madrid: Léeme Libros.

Migelez, X. (2017). Aura Garrido se hace un Rodolfo Sancho en la nueva temporada de 'El Ministerio'. En Vanitatis. Recuperado de 
http://www.vanitatis.elconfidencial.com/television/seriestv/2017-03-24/exclusiva-aura-garrido-baja-temporal-el-ministerio-del-tiempo-temporada3_1354470/

Miranda-Galbe, J. y Figuero-Espadas, J. (2016). El rol del prosumidor en la expansión narrativa transmedia de las historias de ficción en televisión: el caso de El Ministerio del Tiempo. Index Comunicación, 6(2), 115-134.

Oterino, M. (2016). "Alejandro Vega, director de RTVE Digital: "'El Ministerio del Tiempo' sigue por ser un fenómeno social". En FórmulaTV. Recuperado de http://www.formulatv.com/noticias/61416/alejandro-vega-director-rtve-el-ministerio-del-tiempo-fenomeno-social/

Patten, D. (2017). "'Timeless' Ripoff Lawsuit looks to be a thing of the past". En Deadline. Recuperado de http://deadline.com/2017/05/timeless-lawsuit-settled-shawn-ryan-eric-kripke-sony-nbcu-1202103021/

Pimentel, A. (2015). Ministéricos: radiografía de un fenómeno fan televisivo en la era de internet y las redes sociales. En RTVE.es. Recuperadode http://www.rtve.es/television/20150421/ministericos-radiografia-fenomeno-fantelevisivo-era-internet/1133040.shtml

Pimentel, A. (2016). “Tiempo de chupitos”, el capítulo de 'El Ministerio del Tiempo' que no viste. En RTVE.es. Recuperado de http://www. rtve.es/television/20160531/tiempo-chupitos-capitulo-ministerio-del-tiempo-no-viste/1353823.shtm

Prensa RTVE (2016). 'El Ministerio del Tiempo' lanza la primera tienda de 'merchandising' oficial con productos diseñados por los fans. En RTVE.es. Recuperado de http://www.rtve.es/rtve/20160304/ ministerio-del-tiempo-lanza-primera-tienda-merchandising-oficial-productos-disenados-fans/1312903.shtml

Prensa RTVE (2017). El Ministerio del Tiempo presenta su tercera temporada más épica, más aventura y el mismo sentido del humor. En RTVE. 
es. Recuperado de http://www.rtve.es/television/20170512/ministerio-del-tiempo-presenta-su-tercera-temporada-mas-epicamas-aventura-mismo-sentido-del-humor/1544702.shtml

Reijnders, S. (2011). Places of the imagination: Media, tourism, culture. Farnham: Ashgate.

Rodríguez-Mateos, D. y Hernández-Pérez, T. (2015). Televisión social en series de ficción y nuevos roles del documentalista audiovisual: el caso de El Ministerio del Tiempo. Index Comunicación, 6(2), 95-120.

Roig, A. (2013). ¿Fans productores? Una aproximación a la ficción y los films de fans. En A. Roig, D. Aranda y J. Sánchez-Navarro (eds.), Fanáticos: la cultura fan (pp. 65-106). Barcelona: UOC.

Rueda Laffond, J. C. y Coronado Ruiz, C. (2016). Historical science fiction: From television memory to transmedia memory in El Ministerio del Tiempo. Journal of Spanish Cultural Studies, 17(1), 87-101.

Schaaff, A. y Pascual, J. (2016). El tiempo es el que es. Barcelona: Plaza \& Janés.

Scolari, C. A. (2012). Media ecology: Exploring the metaphor to expand the theory. Communication Theory, 22(2), 204-225.

Scolari, C. A. (2013). Narrativas transmedia: cuando todos los medios cuentan. Barcelona: Deusto.

Scolari, C. A. (2015a). Ecología de los medios. Barcelona: Gedisa.

Scolari, C. A. (2015b). Regreso a El Ministerio del Tiempo. En C. Cascajosa Virino (ed.), Dentro de 'El Ministerio del Tiempo' (pp. 227-230). Madrid: Léeme Libros.

Scolari, C. A., Guerrero-Pico, M. y Establés, M. J. (en prensa). Better late than never: Spanish fictional transmedia production. En M. Freeman y W. Proctor (ed.), Transmedia earth. Londres: Routledge. 
Scolari, C. A., Jiménez, M. y Guerrero, M. (2012). Narrativas transmediáticas en España: cuatro ficciones en busca de un destino cross-media. Comunicación y Sociedad, 25(1), 137-163.

Terán, B. (2017). Netflix: así saca partido a 'El Ministerio del Tiempo' (aunque sea una producción real de TVE). En La Información.com. http://blogs.lainformacion.com/telediaria/2017/06/13/netflixasi-saca-partido-a-el-ministerio-del-tiempo-aunque-sea-una-produccion-real-de-tve/

Varona Aramburu, D. y Lara, P. (2015). “Be ministérico, my friend”. Diseño de una estrategia transmedia. En C. Cascajosa Virino (ed.), Dentro de 'El Ministerio del Tiempo' (pp. 203-210). Madrid: Léeme Libros.

Ver Tele (2017). El Ministerio del Tiempo cierra su midseason con mínimo histórico. En Ver Tele. Recuperado de http://vertele.eldiario. es/audiencias-canales/analisis-espana/audiencias6julio-jueveselministerio-midseason-minimo_0_1918908096.html 